\title{
HIDRÓLISE DE CELULOSE POR CATALISADORES MESOESTRUTURADOS NiO-MCM-41 E MoO -MCM-41
}

\author{
Adriano Sant'Ana Silva, Flávio Luiz Honorato da Silva*, Maria Wilma N. Cordeiro Carvalho e Kleberson Ricardo de \\ Oliveira Pereira \\ Unidade Acadêmica de Engenharia Química, Centro de Ciência e Tecnologia, Universidade Federal de Campina Grande, \\ Campus I, Av. Aprígio Veloso, 882, 58429-140 Campina Grande - PB, Brasil
}

Ezenildo Emanuel de Lima

Instituto Federal de Educação, Ciência e Tecnologia de Pernambuco, Campus Afogados da Ingazeira, Sítio Campinhos s/n, 56800-000 Afogados da Ingazeira - PE, Brasil

Recebido em 6/4/11; aceito em 1/11/11; publicado na web em 4/1/12

\begin{abstract}
CELLULOSE HYDROLYSIS BY MESOESTRUTURATED NiO-MCM-41 AND MoO 3 -MCM-41 CATALYSTS. This study was carried out to synthesize, characterize and evaluate the application of mesoestruturated catalysts $\mathrm{MCM}-41,5 \% \mathrm{MoO}_{3}-\mathrm{MCM}-41$ and $5 \% \mathrm{NiO}-\mathrm{MCM}-41$ in the hydrolysis of microcrystalline cellulose. XRD results indicate that the phase of mesoporous MCM-41 was obtained and that the introduction of metal oxides did not affect this mesoporous phase. About the heterogeneous hydrolysis reaction, it was observed that the increase in temperature results in a higher concentration of glucose and the catalyst $5 \% \mathrm{MoO}_{3}-\mathrm{MCM}-41$ provides the highest concentrations of glucose.
\end{abstract}

Keywords: biofuels; mesoporous; glucose.

\section{INTRODUÇÃO}

A procura por fontes de energia renováveis, que sejam alternativas viáveis frente ao petróleo e seu derivados, tem-se propagado cada vez mais na comunidade científica atual. Os combustíveis fósseis e seus derivados, atualmente, atendem a maioria de nossas necessidades, tanto energética quanto quimicamente. Entretanto, a demanda existente por estes combustíveis, em virtude de muitos países tornarem-se industrializados, bem como a elevação no consumo de energia e da população mundial, favorece a uma constante especulação no preço do barril de petróleo.

Somando-se a isso, têm-se os produtos oriundos da queima dos combustíveis fósseis, os quais colaboram diretamente com o aumento de problemas de saúde nas grandes metrópoles. Além disso, sugere-se que o efeito estufa esteja interligado com o aumento na emissão de dióxido de carbono.

Sendo uma tendência global, inúmeras pesquisas estão sendo desenvolvidas no sentido do aproveitamento de recursos renováveis, como óleo vegetal e biomassas, para a produção de biocombustíveis e matérias-primas para a indústria. Entre os mais variáveis recursos disponíveis, as biomassas surgem como uma importante alternativa, não só para desenvolver tecnologias de geração de energia, como também para a produção de outros subprodutos da indústria química, que hoje são produzidos basicamente a partir do petróleo. ${ }^{1}$

A grande vantagem na utilização de produtos derivados da biomassa, desconsiderando-se o necessário desenvolvimento científico e tecnológico, decorre do fato desta apresentar baixo valor agregado e ser abundante. Além disso, resíduos de processos agrícolas, florestais ou industriais, que muitas vezes não teriam uma destinação adequada, podem ser utilizados como fonte de carbono, evitando-se assim prejuízos ambientais face ao desequilíbrio gerado pelo descarte inadequado desse material. ${ }^{1}$

A biomassa pode ser definida como todo material vivo ou morto derivado de plantas, animais e possíveis resíduos provenientes destes. Entretanto, a biomassa das plantas, em especial o amido e os

*e-mail: flavioluizh@yahoo.com.br materiais lignocelulósicos (celulose, hemicelulose e lignina), tem atraído maior interesse para a obtenção de biocombustíveis, como é o caso do etanol celulósico. Todavia, o uso do amido esbarra na sua aplicação na indústria de alimentos, isto é, o uso deste polímero na obtenção de biocombustíveis pode gerar especulação financeira e, também, falta no mesmo no mercado. ${ }^{2}$

Por outro lado, os materiais lignocelulósicos, em especial a celulose, apresentam-se viáveis em virtude de sua disponibilidade e de não ter aplicação direta na indústria de alimentos. Além disso, resíduos de agroindústrias, por exemplo, cana-de-açúcar, bagaço de caju, polpa de sisal, resíduo da indústria de extração de óleo vegetal, etc., antes sem nenhum valor financeiro e fontes certas de poluição, podem ser aproveitados na produção de carboidratos, como glicose, xilose, arabinose, etc. que, posteriormente, podem ser convertidos, por meio de fermentação, em diversos produtos, dentre estes o etanol celulósico.

A celulose é composta de uma série de D-glicose interligada por ligações $\beta-1,4$ glicosídicas, as quais podem ser convertidas em monossacarídeos, neste caso em glicose, que serve como plataforma para a síntese de uma variedade de compostos químicos como etanol celulósico, medicamentos e alimentos. ${ }^{3}$ A conversão da celulose em glicose ocorre pela reação de hidrólise, e essa pode ser catalisada por enzimas biológicas, ácidos minerais (ácido clorídrico, sulfúrico, etc.), catalisadores sólidos (zeólitas, heteropoliácidos, etc.), como também pode ser executada na ausência destes catalisadores como, por exemplo, reações em condições sub ou supercríticas.

Os processos de hidrólise enzimática e de ácidos minerais são os mais utilizados comercialmente e, ainda, apresentam elevados percentuais de conversão em monossacarídeos. Entretanto, embora estes processos sejam mais efetivos, os ácidos minerais causam sérios problemas de poluição, além de comprometerem a integridade física dos equipamentos. ${ }^{4}$ Somado a isso, tem-se o fato dos ácidos minerais não poderem ser reutilizados, e o custo elevado das enzimas, mesmo com a possibilidade de serem imobilizadas, possuem custo elevado.

Como alternativa ao processo com enzimas e ácidos minerais, os catalisadores sólidos, tais como óxidos metálicos de metais de transição, heteropoliácidos, zeólitas, materiais mesoporosos, etc., têm uma 
grande vantagem, por exemplo, podem ser reutilizados, baixo nível de comprometimento dos equipamentos industriais, menor geração de efluentes, o licor obtido não necessita de neutralização, etc.

Diversos estudos sobre hidrólise heterogênea da celulose foram conduzidos visando obter monossacarídeos para fermentação. Tian e colaboradores estudaram a hidrólise da celulose microcristalina na presença do heteropoliácido ácido tungifosfórico para uma faixa de temperatura de 150 a $190{ }^{\circ} \mathrm{C}$, razão celulose:água de 50 e 0,08 mmol de catalisador, com tempo de 1 a 6 h. ${ }^{3}$ Nesse estudo, Tian e seus colaboradores, após $2 \mathrm{~h}$ de reação, obtiveram teores de glicose de 10 a $50 \%$.

Fukuoka e Dhepe estudaram a hidrólise heterogênea da celulose microcristalina na presença dos catalisadores HZSM-5, Hb, HUSY(20), HUSY (30), $\mathrm{SiO}_{2}, \mathrm{y}-\mathrm{Al}_{2} \mathrm{O}_{3}, \mathrm{SiO}_{2}-\mathrm{Al}_{2} \mathrm{O}_{3}$, etc. na temperatura de $190{ }^{\circ} \mathrm{C}$, por $24 \mathrm{~h}$, na presença de $42 \%$ de catalisador e razão celulose:água de $125 .{ }^{5}$ No estudo, o sistema reacional ainda foi pressurizado com hidrogênio gasoso. Constaram que a zeólita HZSM- 5 foi o catalisador com melhor resultado, resultando em cerca de $3,5 \%$ de glicose.

Diante disso, o presente estudo objetivou sintetizar, caracterizar o suporte MCM-41 e os catalisadores $5 \% \mathrm{MoO}_{3}-\mathrm{MCM}-41$ e $5 \% \mathrm{NiO}-$ -MCM-41 e avaliar o desempenho destes na reação de hidrólise da celulose.

\section{PARTE EXPERIMENTAL}

\section{Síntese do suporte e catalisador}

Os reagentes utilizados na síntese do suporte MCM-41 foram o brometo de hexadeciltrimetilamônio (CTMABr) (97\%, Merck), tetraetilo ortossilicato (TEOS) $\left(98 \%, 28 \%\right.$ de $\mathrm{SiO}_{2}$, Merck), amônia em solução ( $25 \%$, Merck) e água deionizada.

O suporte mesoporoso MCM-41 foi sintetizado de acordo com metodologia descrita na literatura, sendo a composição molar do gel de síntese $\mathrm{SiO}_{2} / 0,12 \mathrm{CTMABr} / 2,50 \mathrm{NH}_{4} \mathrm{OH} / 150 \mathrm{H}_{2} \mathrm{O}{ }^{6}$

A calcinação da MCM-41 sintetizada foi realizada em forno tipo mufla a $540{ }^{\circ} \mathrm{C}$, seguindo rampa de aquecimento de $100{ }^{\circ} \mathrm{C} / 1 \mathrm{~h} ; 200$ ${ }^{\circ} \mathrm{C} / 1 \mathrm{~h} ; 350{ }^{\circ} \mathrm{C} / 1 \mathrm{~h}$ e $540{ }^{\circ} \mathrm{C} / 6 \mathrm{~h}$, a taxa de aquecimento de $10^{\circ} \mathrm{C} / \mathrm{min}$.

A síntese dos catalisadores $5 \% \mathrm{MoO}_{3}-\mathrm{MCM}-41$ e $5 \% \mathrm{NiO}-$ -MCM-41 foi realizada empregando-se a técnica de dispersão física. ${ }^{7}$ Para o preparo do catalisador utilizou-se o trióxido de molibdênio $\left(\mathrm{MoO}_{3}\right)(99 \%$ - Vetec) e o óxido de níquel, obtido pela calcinação do nitrato de níquel hexa-hidratado (Merck) por meio da rampa de aquecimento de $100{ }^{\circ} \mathrm{C} / 1 \mathrm{~h} ; 200^{\circ} \mathrm{C} / 1$ h e $350^{\circ} \mathrm{C} / 4 \mathrm{~h} .{ }^{8}$

Previamente à dispersão física, o suporte e os óxidos foram secos em estufa a $150{ }^{\circ} \mathrm{C}$ por $24 \mathrm{~h}$, seguindo rampa de aquecimento de 60 ${ }^{\circ} \mathrm{C} / 3 \mathrm{~h}, 100^{\circ} \mathrm{C} / 1 \mathrm{~h}$ e $150{ }^{\circ} \mathrm{C} / 20 \mathrm{~h}$. Após este procedimento, o suporte e os óxidos secos foram pesados e, em seguida, dispostos em almofariz para serem misturados por $30 \mathrm{~min}$.

A calcinação da mistura óxido:suporte foi realizada em forno tipo mufla a $500{ }^{\circ} \mathrm{C}$ seguindo rampa de aquecimento de $100{ }^{\circ} \mathrm{C} / 0,5 \mathrm{~h} ; 200$ ${ }^{\circ} \mathrm{C} / 0,5 \mathrm{~h} ; 300{ }^{\circ} \mathrm{C} / 0,5 \mathrm{~h} ; 400{ }^{\circ} \mathrm{C} / 0,5$ h e $500{ }^{\circ} \mathrm{C} / 4 \mathrm{~h} .{ }^{7}$

\section{Caracterização do suporte e catalisador}

Os difratogramas de raios- $\mathrm{X}$ do suporte e dos catalisadores foram realizados empregando o método do pó. Os ensaios foram realizados em difratômetro Shimadzu, modelo XRD 600, com fonte de radiação $\mathrm{CuK} \alpha(\lambda=1,5406 \AA$ ), obtida por $40 \mathrm{kV}$ em corrente de filamento de $30 \mathrm{~mA}$. As medidas foram realizadas com o passo de $0,02^{\circ} \mathrm{e} \mathrm{em}$ tempo de $0,6 \mathrm{~s}$.

Os resultados obtidos na análise de DRX foram utilizados na determinação do espaçamento interplanar (Equação 1) e o parâmetro unitário para a rede hexagonal $\left(\mathrm{a}_{0}\right)$ (Equação 2). O espaçamento interplanar $\left(\mathrm{d}_{100}\right)$ foi determinado para os índices de Miller (hkl) referente ao pico de reflexão (100) obtido no difratograma. ${ }^{9}$

$$
\begin{gathered}
d_{100}=\frac{\lambda}{2 \operatorname{sen} \theta} \\
a_{0}=\frac{2 d_{100}}{\sqrt{3}}
\end{gathered}
$$

em que $\lambda$ é o comprimento de onda da radiação $\operatorname{CuK} \alpha(0,15418 \mathrm{~nm}$ ou $15,418 \AA$ ) e $d_{100}$ é a distância interplanar referente ao ponto de reflexão (100).

A análise textural do suporte e dos catalisadores sintetizados foi realizada em analisador de área superficial e tamanho de poro da Quantachrome modelo Nova 3200e. Com a análise foi possível obter as isotermas de adsorção/dessorção das amostras, a área superficial, a área superficial externa, o volume poroso, o diâmetro de poros e sua distribuição e a espessura da parede das amostras. A área superficial foi determinada pelo método de BET, o qual se baseia nos dados de adsorção na faixa de pressão relativa $\left(\mathrm{P} / \mathrm{P}_{0}\right)$ de 0,05 a $0,30 .{ }^{10}$ $\mathrm{O}$ diâmetro de poro foi determinado usando o método de $\mathrm{BJH}$ e o volume poroso pela quantidade de nitrogênio adsorvido a $\mathrm{P} / \mathrm{P}_{0}$ igual a $0,95 .{ }^{11} \mathrm{~A}$ espessura da parede das amostras $(e)$ foi obtida por meio da diferença entre o diâmetro de poro $(D p)$ e o parâmetro unitário de célula $\left(a_{0}\right)\left(e=D p-a_{0}\right){ }^{9}$

A área externa das amostras foi pelo método $\alpha$-plot e como o procedimento exige uma isoterma de adsorção de um material de referência, utilizou-se a Aerosil-200. ${ }^{12,13}$

Para a realização das análises, as amostras foram secas em estufa sem circulação de ar a $150{ }^{\circ} \mathrm{C}$ por $12 \mathrm{~h}$ e, posteriormente, sob vácuo no equipamento, a $300^{\circ} \mathrm{C}$ por $6 \mathrm{~h}$.

Os espectros de infravermelho por transformada de Fourier (FTIR) foram obtidos em espectrômetro Nicolet modelo Avatar-369. As pastilhas foram obtidas mediante diluição das amostras, previamente secas em estufa a $110{ }^{\circ} \mathrm{C}$ por $3 \mathrm{~h}$, em brometo de potássio (KBr) na concentração de $2 \%$ em massa.

\section{Testes catalíticos}

Os testes catalíticos foram conduzidos em autoclaves de politetrafluoretileno encamisadas por peças de aço inox, sendo as reações conduzidas a 160,180 e $200^{\circ} \mathrm{C}$, com razão mássica de celulose:água de 1:10, percentual de catalisador de $5 \%$ e tempo de reação de $3 \mathrm{~h}$. Para cada reação utilizou-se $1 \mathrm{~g}$ de celulose microcristalina (Vetec) e $10 \mathrm{~g}$ de água deionizada e, objetivando avaliar a atividade dos óxidos metálicos introduzidos ao suporte sobre a hidrólise, foram conduzidas reações sem catalisador.

Ao término dos testes catalíticos o produto reacional foi filtrado a vácuo e o sobrenadante (licor) obtido foi analisado quanto ao teor de glicose $\left(\mathrm{mg} \mathrm{L}^{-1}\right)$ por cromatografia líquida de alta eficiência (CLAE). O equipamento consiste de uma bomba ProStar 210 (Varian; vazão de $0,6 \mathrm{~mL} \mathrm{~min}^{-1}$; fase móvel água miliQ) acoplada a um detector de índice de refração, coluna analítica de aço inox Hi-Plex (Varian; $300 \times 7,7 \mathrm{~mm})$.

\section{RESULTADOS}

Na Figura 1 encontram-se os difratogramas de raios-X da MCM41, não calcinada e calcinada, e dos catalisadores $5 \% \mathrm{MoO}_{3}-\mathrm{MCM}-41$ e $5 \% \mathrm{NiO}-\mathrm{MCM}-41$. Conforme pode ser observado, a presença dos três pontos de reflexão (100), (110) e (200) para o MCM-41 não calcinado e calcinado indica a formação da estrutura de simetria hexagonal do tipo p6mm pertencente a MCM-41..$^{6,14}$ 


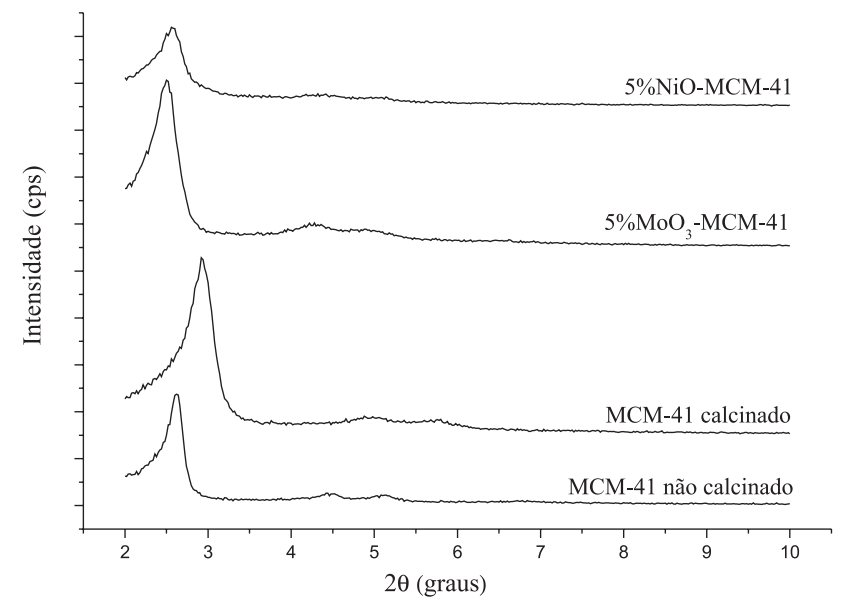

Figura 1. Difratograma de raios-X da MCM-41 não calcinada e calcinada e dos catalisadores sintetizados

A análise dos resultados revela ainda que o processo de dispersão física do $\mathrm{MoO}_{3}$ e NiO no suporte mesoporoso MCM-41 não comprometeu severamente a estrutura hexagonal do material, mesmo após o tratamento térmico (calcinação).

Na Figura 2 os difratogramas dos catalisadores $5 \% \mathrm{MoO}_{3}$ -MCM-41 e 5\%NiO-MCM-41, para a faixa de ângulo (20) de 10 a $90^{\circ}$, são apresentados. Os resultados desta figura indicam uma distribuição uniforme de $\mathrm{MoO}_{3}$ sobre a superfície do suporte, o que é evidenciado pela ausência da fase deste óxido. A presença da ampla banda na faixa de ângulo de 10 a $35^{\circ}$ é referente ao óxido de silício $\left(\mathrm{SiO}_{2}\right)$ amorfo presente na parede da MCM-41. ${ }^{15}$

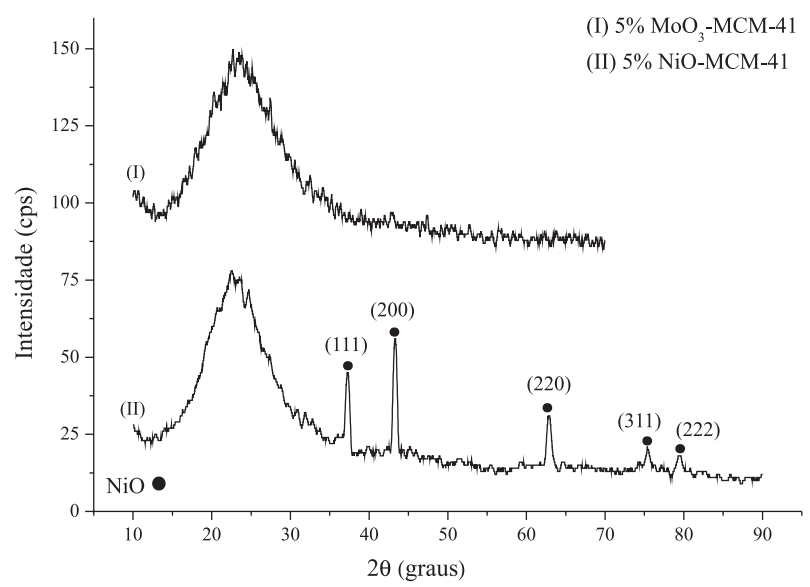

Figura 2. Difratogramas de raios- $X$ dos catalisadores sintetizados no estudo

Contrariamente aos catalisadores preparados com $\mathrm{MoO}_{3}$, foi observada a presença da fase do óxido de níquel cúbico de face centrada, na faixa de ângulo de 10 a $90^{\circ}$ (Figura 2). Conforme a biblioteca do International Center for Diffractional Data (JCPDS) (Registro JCPDS: 73-1523) foram identificados picos (20) em 37,23; 43,25; 62,$83 ; 75,35$ e 79,34 .

A presença da fase $\mathrm{NiO}$ é um indicativo de que parte do óxido não foi completamente dispersa sobre o suporte após a calcinação, ou seja, permaneceu na parte externa do suporte. ${ }^{16,17}$

Na Figura 3 são apresentados os espectros de FTIR do suporte não calcinado, do suporte calcinado e dos catalisadores sintetizados. A análise destes resultados revela a presença dos íons hexadeciltrimetilamônio $\left(\mathrm{CTMA}^{+}\right)$nas bandas vibracionais de 960, 1482, 2852 e $2916 \mathrm{~cm}^{-1}$. Em $960 \mathrm{~cm}^{-1}$ foi observado o estiramento assimétrico da ligação $\mathrm{CH}_{3}-\mathrm{N}^{+}$do agrupamento polar do $\mathrm{CTMA}^{+}$. A banda vibracio- nal de $1482 \mathrm{~cm}^{-1}$ refere-se às deformações do íon $\mathrm{CTMA}^{+} \mathrm{e}$ em 2852 e $2916 \mathrm{~cm}^{-1}$ foram observados os estiramentos entre as ligações $\mathrm{C}-\mathrm{H}$ dos grupos $\mathrm{CH}_{2}$ e $\mathrm{CH}_{3}$ do íon CTMA ${ }^{+18,19}$

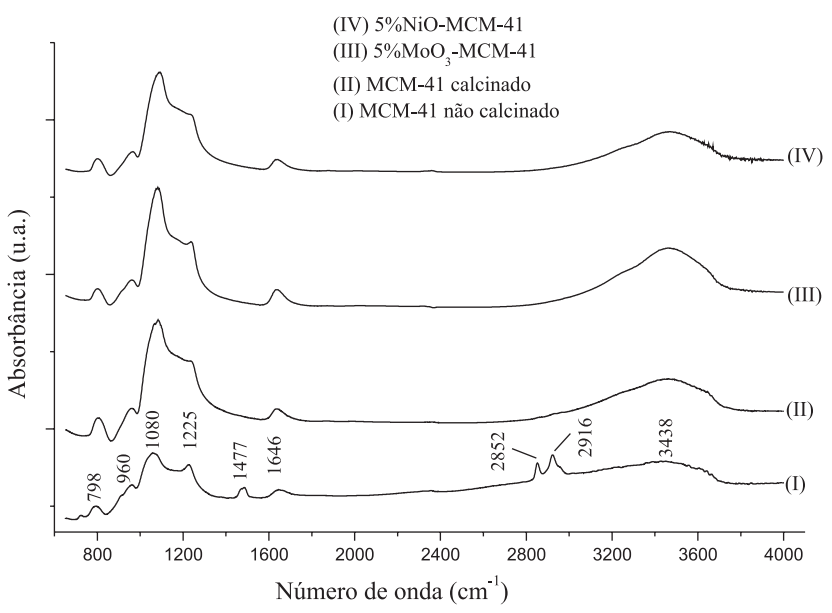

Figura 3. Espectros de FTIR do suporte MCM-41 não calcinado e calcinado

Os resultados na Figura 3 indicam a remoção do direcionador brometo de hexadeciltrimetilamônio (CTMABr), o que é observado pela ausência das bandas vibracionais em 1482, 2852 e $2916 \mathrm{~cm}^{-1}$. Quanto à banda vibracional de $960 \mathrm{~cm}^{-1}$, constatou-se redução na intensidade desta após a calcinação.

$\mathrm{Na}$ banda vibracional de $1080 \mathrm{~cm}^{-1}$, foram observadas as vibrações das ligações $\mathrm{Si}-\mathrm{O}-\mathrm{Si}$ do tetraedro $\mathrm{SiO}_{4}$, referentes à estrutura mesoporosa, e em $1225 \mathrm{~cm}^{-1}$ foi observado o estiramento assimétrico associado à ligação $\mathrm{Si}-\mathrm{O} .{ }^{20} \mathrm{~A}$ presença de água e do grupo de hidroxilas interno e externo da estrutura mesoporosa foi verificada pelas bandas vibracionais de 1646 e $3438 \mathrm{~cm}^{-1}$ para a MCM-41 não calcinada e em 1638 e $3449 \mathrm{~cm}^{-1}$ para a MCM-41 calcinada. $^{21}$

Quanto às ligações Si-O da estrutura hexagonal mesoporosa da MCM-41 verificou-se para o material não calcinado e o calcinado a presença do estiramento assimétrico desta ligação, nas respectivas bandas vibracionais de 455 e $449 \mathrm{~cm}^{-1}$. Por outro lado, em 798 e $801 \mathrm{~cm}^{-1}$, constatou-se a presença das ligações simétricas Si-O das amostras não calcinadas e calcinadas, respectivamente. ${ }^{18}$

Constatou-se ainda que, após a calcinação do suporte, houve o deslocamento da banda vibracional 1080 para $1077 \mathrm{~cm}^{-1}$. O deslocamento excessivo para posições próximas da sílica amorfa, cerca de $1115 \mathrm{~cm}^{-1}$, poderia indicar que o processo de calcinação foi acompanhado de um colapso estrutural gerando espécies de sílica amorfa a partir de estruturas de sílica organizadas tipo MCM-41. ${ }^{19}$, ${ }^{22}$ Portanto, como se pode observar, não houve um deslocamento da banda vibracional para posições próximas à da sílica, o que sugere a não ocorrência do colapso.

Os resultados constantes na Figura 3 indicam ainda que os espectros de FTIR dos catalisadores sintetizados apresentam o mesmo perfil do suporte MCM-41 calcinado. Entretanto, ao se ampliar a faixa do espectro de 600 a $1200 \mathrm{~cm}^{-1}$ (Figura 4) verificou-se a presença das bandas vibracionais em 797, 914, 964 e $1047 \mathrm{~cm}^{-1}$.

A banda vibracional observada em $914 \mathrm{~cm}^{-1}$ refere-se à vibração da ligação Mo-O-Si presente no molibdato tetraédrico ligado à superfície do suporte. Além disso, com o aumento da concentração de $\mathrm{MoO}_{3}$, houve acréscimo na intensidade desta banda vibracional. $\mathrm{Na}$ banda vibracional de $964 \mathrm{~cm}^{-1}$ verificou-se a presença do grupo $\mathrm{Si}=\mathrm{O}$ na superfície da fase do molibdato..$^{23,24}$

A banda vibracional em $964 \mathrm{~cm}^{-1}$, normalmente, é atribuída ao estiramento da ligação Si-O-H. Entretanto, foi observado um aumento 


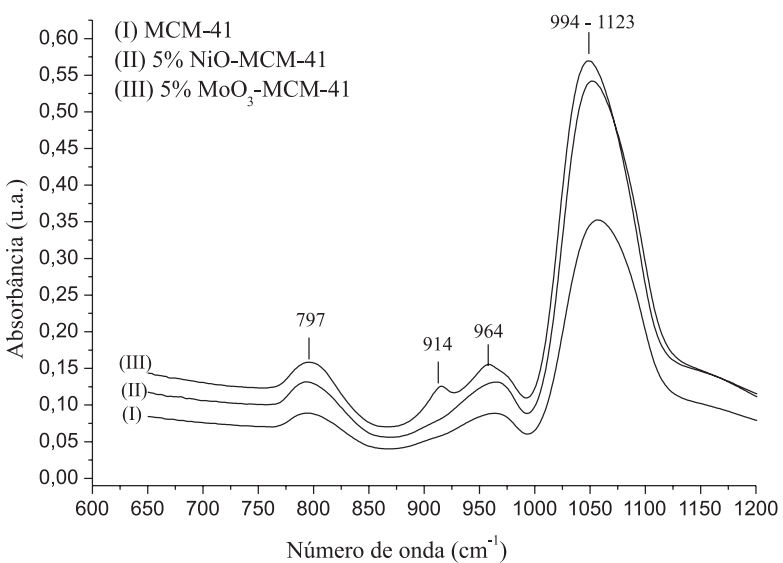

Figura 4. Espectros de FTIR do suporte calcinado e não calcinado e dos catalisadores sintetizados

na intensidade desta banda vibracional, indicando a presença de $\mathrm{MoO}_{3}$ na estrutura da MCM-41.25

De acordo com a literatura, a presença de $\mathrm{MoO}_{3}$ na estrutura da MCM-41, normalmente, é evidenciada por meio das bandas vibracionais em 915, 960 e $994 \mathrm{~cm}^{-1} .{ }^{26}$ Entretanto, no presente estudo, a banda vibracional em $994 \mathrm{~cm}^{-1}$, que se refere ao estiramento vibracional $\mathrm{Si}=\mathrm{O}$ de $\mathrm{MoO}_{3}$, não foi observada, e tal constatação pode estar relacionada à sobreposição da banda vibracional referente à ligação Si-O-Si e ao estiramento de superfície do grupo silanol (Si-OH). As bandas vibracionais em 797 e $1043 \mathrm{~cm}^{-1}$ referem-se, respectivamente, aos estiramentos simétricos e assimétricos da ligação Si-O-Si. ${ }^{27}$

Com relação aos espectros de FTIR do catalisador NiO-MCM-41, verificou-se o mesmo perfil observado para o catalisador $\mathrm{MoO}_{3}$. -MCM-41. A vibração da ligação Ni-O somente é observada na banda vibracional em torno de 440 e $460 \mathrm{~cm}^{-1}$, e isto não foi verificado no presente estudo em virtude da limitação de varredura do equipamento utilizado ser de 600 até $4000 \mathrm{~cm}^{-1} .^{28}$

Na Figura 5 as isotermas de adsorção do suporte e dos catalisadores $5 \% \mathrm{MoO}_{3}-\mathrm{MCM}-41$ e 5\% NiO-MCM-41 encontram-se apresentadas, respectivamente. Pode-se observar em todos os casos a presença de isotermas do tipo IVc e que a introdução dos óxidos na estrutura do suporte não comprometeu o perfil observado. ${ }^{29}$

$\mathrm{Na}$ Tabela 1 os resultados referentes ao espaçamento interplanar $\left(\mathrm{d}_{100}\right)$, ao parâmetro unitário da rede hexagonal $\left(\mathrm{a}_{0}\right)$, área superficial, área superficial externa, volume poroso, diâmetro de poro e espessura da parede do suporte e dos catalisadores sintetizados encontram-se apresentados.

Os resultados revelam (Tabela 1) o deslocamento de $\mathrm{d}_{100}$ do suporte quando calcinado, de $2,140^{\circ}$ para $2,299^{\circ}$. Paralelamente ao deslocamento deste pico, verificou-se uma contração da estrutura hexagonal do suporte, o que é observado pela redução de $\mathrm{a}_{0}$ e de $\mathrm{d}_{100}$. A contração observada após a calcinação do suporte está relacionada com a reorganização dos íons $\mathrm{Si}^{-} \mathrm{O}^{-}$e a condensação do grupo sila-

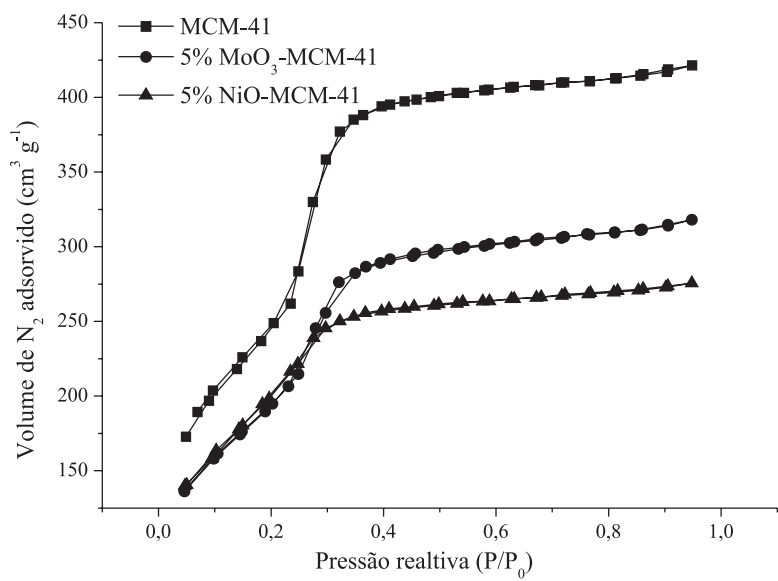

Figura 5. Isotermas de adsorção (a) e distribuição dos poros (b) para o suporte e catalisadores sintetizados

nol (三Si-OH) devido à remoção do agente direcionador brometo de cetiltrimetilamônio (CTMABr).

Com a remoção do CTABr, que desempenha função mantenedora da estrutura, ocorre um desequilíbrio na tensão superficial da parede interna do poro, a qual tende a levar o sistema a se contrair fisicamente. Além disso, a remoção do direcionador permite que os grupos $\mathrm{Si}^{-} \mathrm{O}^{-}$se reorganizem formando novas ligações entre siloxanas, como também os grupos $\mathrm{Si}-\mathrm{OH}$ se condensam de modo a restabelecer o equilíbrio. Assim, há um aumento no ordenamento do sistema poroso, aumentando a intensidade dos picos, e uma contração do parâmetro de rede hexagonal, deslocando o ângulo dos picos para valores maiores..$^{30,31}$

Quanto aos catalisadores preparados (Tabela 1), verifica-se aumento nos valores de $\mathrm{d}_{100}$ e $\mathrm{a}_{0}$, quando comparados com o suporte, o que indica a difusão dos óxidos para o interior dos poros do suporte ao término da calcinação. Entretanto, os valores de $\mathrm{d}_{100}$ e $\mathrm{a}_{0}$ para o catalisador $\mathrm{MoO}_{3}-\mathrm{MCM}-41$, nas concentrações em estudo, foram superiores aos de NiO-MCM-41, sugerindo assim que a incorporação do trióxido de molibdênio, nos poros da MCM-41, foi maior em relação aos catalisadores preparados com óxido de níquel, em média $2,5 \%$ superior. ${ }^{32,33}$

Foi observada também redução de área superficial, área superficial externa, volume poroso e diâmetro de poro com a introdução de $\mathrm{NiO}$ e $\mathrm{MoO}_{3}$ no suporte. A redução observada na área superficial para os catalisadores $5 \% \mathrm{NiO}-\mathrm{MCM}-41$ e $5 \% \mathrm{MoO}_{3}-\mathrm{MCM}-41$ pode estar relacionada com a perda da cristalinidade como material, o que é observado pela redução na intensidade $\mathrm{d}_{100}$, e ao processo de difusão dos óxidos metálicos sobre a superfície do suporte. ${ }^{27,34,35}$

Poucos óxidos podem ser dispersos espontaneamente na superfície de um suporte, durante um tratamento térmico, com a formação de uma monocamada ou submonocamada, por exemplo, uma fase tridimensional do óxido metálico se transforma em bidimensional na superfície do suporte. ${ }^{36}$ Portanto, ao se analisar a área superficial externa verificou-se que a maior área para o catalisador $5 \% \mathrm{MoO}_{3}$ -MCM-41, quando comparado com o $5 \% \mathrm{NiO}-\mathrm{MCM}-41$, pode

Tabela 1. Resultados obtidos da caracterização textural do suporte e dos catalisadores

\begin{tabular}{lccccccc}
\hline \multicolumn{1}{c}{ Amostras } & $\mathrm{d}_{100}(\mathrm{~nm})$ & $\mathrm{a}_{0}(\mathrm{~nm})$ & $\mathrm{A}_{\mathrm{BET}}\left(\mathrm{m}^{2} \mathrm{~g}^{-1}\right)$ & $\mathrm{A}_{\mathrm{EXT}}\left(\mathrm{m}^{2} \mathrm{~g}^{-1}\right)$ & $\mathrm{Vp}\left(\mathrm{cm}^{3} \mathrm{~g}^{-1}\right)$ & $\mathrm{Dp}(\mathrm{nm})$ & - \\
\hline MCM-41 não calcinada & 3,405 & 3,932 & - & - & - & - & - \\
MCM-41 calcinada & 3,202 & 3,697 & 1119,0 & 36,953 & 0,652 & 2,431 & 1,266 \\
$5 \%$ MoO & -MCM-41 & 3,554 & 4,104 & 771,1 & 35,709 & 0,442 & 2,343 \\
$5 \%$ NiO MCM-41 & 3,468 & 4,005 & 652,0 & 25,459 & 0,426 & 2,343 & 1,761 \\
\hline
\end{tabular}

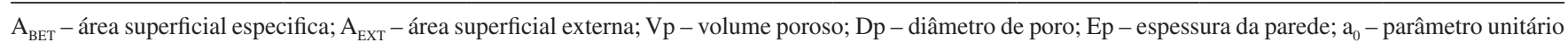
de célula; $\mathrm{d}_{100}-$ espaçamento interplanar 
estar relacionada com esta capacidade de difusão do óxido sobre a superfície do suporte.

Na Figura 6 são apresentados os resultados da hidrólise heterogênea da celulose microcristalina para os catalisadores em estudo. Estes resultados indicam dependência direta com a variável temperatura, ou seja, a concentração da glicose aumenta proporcionalmente com a elevação da temperatura.

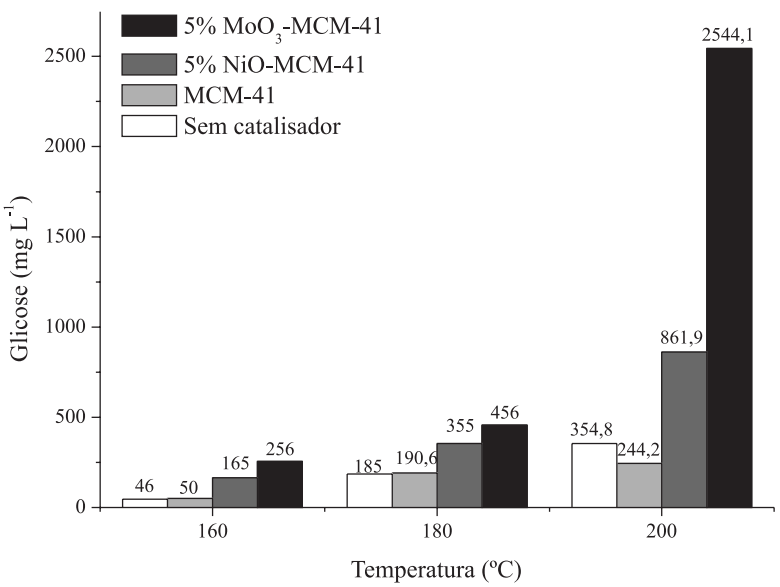

Figura 6. Resultados dos testes catalíticos da hidrólise heterogênea da celulose microcristalina

Outro ponto importante a ser observado refere-se ao fato de que para as reações conduzidas na presença dos catalisadores, a concentração de glicose foi superior às reações sem catalisador e com o suporte. Esta observação indica que os catalisadores $5 \% \mathrm{NiO}$ -MCM-41 e 5\% $\mathrm{MoO}_{3}-\mathrm{MCM}-41$ apresentam atividade catalítica sobre a reação de hidrólise, e que o último citado foi o que resultou nas maiores concentrações de glicose. A superioridade do catalisador $5 \% \mathrm{MoO}_{3}-\mathrm{MCM}-41$ frente a $5 \% \mathrm{NiO}-\mathrm{MCM}-41$ pode estar relacionado com a maior predominância dos forte sítios ácidos de Brönsted. ${ }^{37,38}$

Diversos estudos de hidrólise heterogênea da celulose microcristalina já foram conduzidos visando determinar um catalisador com maior atividade neste processo reacional. Lai e colaboradores avaliaram os catalisadores $\mathrm{Fe}_{3} \mathrm{O}_{4}-\mathrm{SBA}-\mathrm{SO}_{3} \mathrm{H}, \mathrm{AC}-\mathrm{SO}_{3} \mathrm{H}$ (carvão ativado sulfonado), Amberlyst-15, $\gamma-\mathrm{Al}_{2} \mathrm{O}_{3}$ e SBA-15 na hidrólise de celulose microcristalina. ${ }^{39}$ Neste estudo os autores obtiveram rendimentos em glicose $26,21,15,3$ e menor que $1 \%$, respectivamente. A reação foi conduzida a $150{ }^{\circ} \mathrm{C}$ com razão mássica celulose:água de $1: 15$, tempo de reação de $3 \mathrm{~h}, 100 \%$ de catalisador em relação à massa de celulose e sob agitação. Embora os resultados obtidos pelos pesquisadores sejam superiores, de maneira geral, ao rendimento médio obtido neste estudo, cerca de $2,5 \%$ para o catalisador $5 \% \mathrm{MoO}_{3}-\mathrm{MCM}-41$, a quantidade de catalisador utilizada no referido estudo ${ }^{39}$ foi cerca de 33 vezes superior e o processo foi conduzido na presença de agitação.

Onda e colaboradores, ao estudarem a hidrólise da celulose microcristalina com os catalisadores $\mathrm{AC}_{-} \mathrm{SO}_{3} \mathrm{H}, \mathrm{SO}_{4}{ }^{2-}-\mathrm{Zr}_{2} \mathrm{O}$, Amberlyst-15, verificaram rendimentos em glicose de 40,5; 25,5 e 14,2\%, respectivamente. Contudo, os experimentos foram conduzidos a 150 ${ }^{\circ} \mathrm{C}$, razão mássica de celulose:água de 1:100, tempo de 24 h, $110 \%$ de catalisador e com agitação. ${ }^{40}$ Além disso, vale destacar que os autores pulverizaram a celulose microcristalina em moinho de bolas orbital, com a finalidade de reduzir a cristalinidade desta, tornando-a mais amorfa facilitando a sua hidrólise.

Foi verificado, neste estudo, que o emprego dos catalisadores heterogêneos na obtenção de açúcares fermentescíveis apresenta viabilidade tecnológica, porém exige a avaliação futura de distintas fontes de biomassas e o desenvolvimento e otimização do processo reacional.

\section{CONCLUSÃO}

Um novo catalisador foi apresentado para a hidrólise de materiais lignocelulósicos. O método de dispersão física empregado no preparo do catalisador dos óxidos metálicos $\mathrm{MoO}_{3}$ e $\mathrm{NiO}$ sobre o suporte MCM-41 pode ser utilizado no preparo de catalisadores com aplicação na hidrólise heterogênea da celulose microcristalina. $\mathrm{O}$ catalisador $5 \% \mathrm{MoO}_{3}-\mathrm{MCM}-41$ apresentou o melhor desempenho na hidrólise da celulose microcristalina.

\section{REFERÊNCIAS}

1. Bevilaqua, D. B.; Dissertação de Mestrado, Universidade Federal de Santa Maria, Brasil, 2010.

2. Dephe, P. L.; Fukuoka, A.; ChemSusChem. 2008, 1, 969.

3. Tian, J.; Wang, J.; Zhao, S.; Jiang, C.; Zhang, X.; Wang, X.; Cellul. 2010, 17, 587.

4. Lange, J. P.; van De Graaf, W. D.; Haan, R. J.; ChemSusChem. 2009, 2 , 437.

5. Fukuoka, A.; Dhepe, P. L.; Ang. Chem., Int. Ed. 2006, 118, 5285.

6. Gaydhankar, T. R.; Samuel, V.; Jha, R. K.; Kumar, R.; Joshi, P. N.; Mater. Res. Bull. 2007, 42, 1473.

7. Félix, C. R. O.; Dissertação de Mestrado, Universidade Federal de Campina Grande, Brasil, 2009.

8. Xiao, F. S.; Zheng, S.; Sun, J.; Yu, R.; Qiu, S.; Xu, R.; J. Catal. 1998, 176, 474.

9. Beck, J. S.; Vartuli, J. C.; Roth, W. J.; Leonowicz, M. E.; Kresge, C. T.; Schmitt, K. D.; Chu, C. T. W.; Olson, D. H.; E. W. Sheppard.; J. Am. Chem. Soc. 1992, 114, 10843.

10. Brunauer, S.; Emmett, P. H.; Teller, E.; J. Am. Chem. Soc. 1938, 60, 309.

11. Barrett, E. P.; Joiyner, L. G.; Halenda, P. P.; J. Am. Chem. Soc. 1953, 73, 373.

12. Condon, J. B.; Surface area and porosity determinations by physisorption: measurements and theory, $1^{\text {st }}$ ed., Elsevier: Oxford, 2006.

13. Frenhani, G. N.; Dissertação de Mestrado, Universidade de Campinas, Brasil, 2002.

14. Mody, H. M.; Kannan, S.; Bajaj, H. C.; Manu, V.; Jasra, R. V.; J. Porous Mater. 2008, 15, 571

15. Martín-Aranda, R. M.; Cejka, J.; Top. Catal. 2010, 53, 141.

16. Lensveld, D. J.; Mesu, J. G.; van Dillen, J.; Jong, P.; Microporous Mesoporous Mater. 2001, 44-45, 401.

17. El-Safty, S. A.; Kiyozumi, Y.; Hanaoka, T.; Mizukami, F.; Appl. Catal., B 2008, 82, 169 .

18. Kaya, E.; Oktar, N.; Karakas, G.; Mürtezaoglu, K.; Turk. J. Chem. 2010, 34, 935.

19. Chen, L. Y.; Jaenicke, S.; Chuah, G. K.; Microporous Mater. 1997, 12, 323.

20. Sousa, A.; Dissertação de Mestrado, Centro de Desenvolvimento da Tecnologia Nuclear, Brasil, 2006.

21. Selvaraj, M.; Pandurangan, A.; Seshadri, K. S.; Sinha, P. K.; Lal, K. B.; Appl. Catal., A 2003, 242, 347.

22. Souza, M. J. B.; Tese de Doutorado, Universidade Federal do Rio Grande do Norte, Brasil, 2005.

23. Thanabodeekij, N.; Gulari, E.; Wongkasemjit, S.; Powder Technol. 2007, 173, 211.

24. Li, Z.; Gao, L.; Zheng, S.; Appl. Catal., A 2002, 236, 163.

25. Cho, D. H.; Chang, T. S.; Ryu, S. K.; Lee, Y. K.; Catal. Lett. 2000, 64, 227.

26. Kostova, N. G.; Kraleva, E.; Spojakina, A. A.; Godocikova, E.; Balaz, P.; J. Mater. Sci. 2007, 42, 3321.

27. Rana, R. K.; Viswanathan, B.; Catal. Lett. 1998, 52, 25.

28. Davar, F.; Fereshteh, Z.; Salavati-Niasari, M.; J. Alloys Compd. 2009, 476, 797. 
29. Rouquerol, F.; Rouquerol, J.; Sing, K.; Adsorption by powders and porous solids: principles, methodology and applications. $1^{\text {st }}$ ed., Academic Press: San Diego, 1999.

30. Sousa, B. V.; Tese de Doutorado, Universidade Federal de Campina Grande, Brasil, 2009.

31. Yu, J.; Shi, J. L.; Wang, L. Z.; Ruan, M. L.; Yan, D. S.; Ceram. Int. 2000, 26,359 .

32. Sugiyama, S.; Kato, Y.; Wada, T.; Ogawa, S.; Nakagawa, K.; Sotowa, K.; Top. Catal. 2010, 53, 550.

33. Li, Z.; Cheng, B.; Su, K.; Gu, Y.; Xi, P.; Guo, M.; J. Mol. Catal. A: Chem. 2008, 289, 100.

34. Debecker, D. P.; Stoyanova, M.; Rodemerck, U.; Eloy, P.; Léonard, A.; Su, B.; Gaigneaux, E. M.; J. Phys. Chem. C 2010, 114, 18664.
35. Knözinger, H.; Taglauer, E. Em Preparation of solid catalyst; Ertl, G.; Knözinger, H.; Weitkamp, J., eds.; Wiley-VCH: Weinhein, 1999, cap. 4.

36. Braun, S.; Appel, L. G.; Camorin, V. L.; Schmal, M.; J. Phys. Chem. B 2000, 104, 6584.

37. Hsu, C. H.; Wang, Y. L.; Ko, A. N.; J. Chin. Chem. Soc. (Taipei, Taiwan). 2009, 56, 908 .

38. Umbarkar, S. B.; Biradar, A. V.; Mathew, S. M.; Shelke, S. B.; Malshe, K. M.; Patil, P. T.; Dagde, S. P.; Niphadkar, S. P.; Dongare, M. K.; Green Chem. 2006, 8, 448.

39. Lai, D.; Deng, L.; Li, J.; Liao, B.; Guo, Q.; Fu, Y.; ChemSusChem. 2011, 4,55 .

40. Onda, A.; Ochi, T.; Yanagisawa, K.; Top. Catal. 2009, 52, 801. 